\title{
Laser Effects on Skin Melanin
}

\author{
Khalid M. Omar \\ Universiti Sains Malaysia, School of Physics \\ 11800 Penang, Malaysia \\ E-mail: khalhadithi@yahoo.com \\ Khaled A. Al-Khaza'leh, M.S. Jaafar, Y. JIDIN \& N. N. Bidi \\ Universiti Sains Malaysia, School of Physics
}

11800 Penang, Malaysia

\begin{abstract}
Melanin is investigated as a pigment that is responsible for colorization in human skin. The color of human skin varies from dark brown to nearly colorless "pinkish white". The amount of skin melanin in the individual skin depends on both genetic and environmental factors. This offers some protection against the damaging effects of ultraviolet radiation. For the studies of laser effects on the skin melanin, the suitable sample is chosen depends on the availability for getting the sample and also the existence of skin melanin on that sample. Therefore, the skin of laboratory rat is used as an experimental sample. Carbon dioxide laser is used as a source of radiation in this study because of its $10.6 \mu \mathrm{m}$ of wavelength which has an ability to penetrate effectively on soft tissue that contains $90 \%$ of water. The rat skin is exposed to carbon dioxide laser " $\mathrm{CO}_{2}$ laser" radiation and any changes on the skin is observed and analyzed. After the exposure, the pieces of normal and exposed skin are preserved. In order to go through the sample, histological method is carried out to make the standard slides for examination under the compound light microscope. The images obtained on the both skin sample the normal and the exposed skin show that there are changes in both skin structures which can be seen clearly under the microscope lenses. These changes contributed by the interaction of the skin cells to the heat and energy produced by the carbon dioxide laser " $\mathrm{CO}_{2}$ laser" during the exposure time.
\end{abstract}

Keywords: Laser application, Medical physics, Laser-tissue interaction

\section{Introduction}

The facts have shown that the theories in physics, chemistry, and mathematics are bonded together in the laser invention. The biological sciences also related to this invention due to the effect of laser beam to the environment. Furthermore, since the invention of the laser in 1960's [Blois, M.C., Quevedo, W.C. et al. (1969), Grossman, K. (2008) ], The unusual properties of laser beam were all too obvious and yet it was not clear in how they could be put to practical use. More and more applications were discovered as the years passed and this attitude slowly changed year by year. There was scarcely an area of sciences and technology in which lasers are utilized for such diverse purposes as aiming missiles, for eyes surgery, for monitoring pollution, for welding, cutting and marking, for checking out goods at supermarket and also for light show entertainment [Blois, M.C., Quevedo, W.C. et al. (1969), Andrews, D.L, (1986)]. All application is taking the advantage of the wavelength of laser that can be used. Different wavelength of lasers will give the different effect on the target tissue. In such medical application, the laser is ordinarily used for treatment or therapy purpose such as cancer or tumor treatment. The use of high-intensity light can destroy the cancer cells. This technique is often used to relieve symptoms of cancer such as bleeding or obstruction, especially when the cancer cannot be cured by other treatments. Lasers can be used in two ways to treat cancer either by shrinking or destroying a tumor with heat, or by activating a chemical that destroys cancer cells [Parks, J. et al (2002)]. For this purpose, the laser that usually used is carbon dioxide laser or Nd:YAG laser which has the wavelength of $10.6 \mu \mathrm{m}$ and $1064 \mathrm{~nm}$ respectively [C. Dierickx, C. et al (2004)]. Due to those wavelengths, they may be used with flexible endoscopes fitted with fiber optics, tubes that allow physicians to see into certain areas of the body. In medical application, laser is commonly used in surgery and treatment procedures on the skin. The most ordinary surgery are like hair removal [Parks, J. et al (2002), C. Dierickx, C. et al (2004), Aesthetic Laser Concepts (2007)], skin resurfacing, treating scars, treating tattoo pigment (tattoo removal) and treatment for tumor cells. The $\mathrm{CO}_{2}$ laser emits spectral energy in the far infra red portion of the electromagnetic spectrum at " $10,600 \mathrm{~nm}$ ". At this wavelength, energy is heavily absorbed by water, which is the primary constituent and chromophore of cells in living tissue. Thus, the energy generated by this laser can be used for cutting or volume ablation by means of tissue vaporization [Lee, S. (2008)]. This unique characteristic 
makes the $\mathrm{CO}_{2}$ laser the most widely used medical laser today. This laser offers the advantages such as pain reduction, swelling reduction, control of infection and minimal surgical bleeding [Long Beach Animal Hospital (2007)].

\section{Methodology}

\subsection{Interaction between $\mathrm{CO}_{2}$ Laser and the Rat Skin}

At " $3 \%$ of PWM", the average of power output generated by the laser is " $2.96 \mathrm{~W}$ " and the power density is " 30.77 $\mathrm{W} / \mathrm{cm}^{2}$ ". At this power output level, the changes on the skin surface are clearly seen by naked eyes. Since the melanin is responsible on the skin coloration, there will be some effect on that soft tissue.

\subsection{Measuring the thickness of dermis layer of the normal skin}

The thickness of the dermis is measured automatically by the software provided for the compound light microscope. The dermis layer of rat skin with 40 times of magnification as shown in "Figure 1". The measurement of the thickness of dermis layer is done on five different places. It gives the average thickness of dermis layer obtained from "Table 1" is " $985.03 \mu \mathrm{m}$ ".

\subsection{Measuring the thickness of epidermis layer of the normal skin}

The epidermis thickness is measured from the upper of black layer to the lower one. This is automatically done by software provide with the light compound microscope. The part of epidermis layer that is being measured under the magnification of 100 times as shown in "Figure 2". From "Table 2", the average thickness of epidermis layer obtained is " $44.80 \mu \mathrm{m}$ ".

\section{Results and Discussion}

The reaction of the skin to laser radiation depends on many factors. The color or amount of pigmentation in the skin is very important in determining the amount of damage that will be produced by a given laser pulse. The output characteristics of the laser, particularly the wavelength are also important. The reactions of the skin to laser radiation vary depending on the exact circumstances and the type of skin that is impossible to specify reactions of the skin exactly. Furthermore, the transmission of radiation through skin varies with the thickness of the different layers of the epidermis and therefore with location on the body. The loss of transmission is due to scattering by the optically discontinuous surfaces in the epidermis, reflection from the surface and the various pigments of the skin and absorption by protein, nucleic acids, carotenoid and hemoglobin pigments. Melanin granules contribute to the absorption and scattering of visible and infra red radiation as well as absorption by ultraviolet radiation. The stratum corneum which is in epidermis layer strongly absorbs far-infrared radiation. Melanin granules are small " $1 \mu \mathrm{m}$ diameter" and not only protect the dermis by absorption of ultraviolet radiation, but also by scattering optical radiation. Melanin scatters rather than absorbs radiation in the near-infrared region. For this and other reasons, near-infrared radiation penetrates deeply into the tissue. Since the index of refraction of the stratum corneum is about 1.5, the Fresnel reflective component is similar to the glass. Optical radiation incident on the skin at grazing angles of incidence is hardly absorbed at all. The relative effectiveness of optical radiation in penetrating the epidermis "and dermis" varies approximately as the cosine of the angle of incidence since light penetrates the outermost layers of the skin and undergoes multiple scattering, and some light is scattered back out of the skin. Since the carbon dioxide laser operates in the far infrared spectral region, it can be categorized as a non-ionizing radiation which has lower energy levels and longer wavelength. Visible light can be seen and feel the burning effects of infrared radiation. Non-ionizing radiation is strong enough to influence the atoms it contacts, but not strong enough to affect their structure.

The heating of skin by infrared radiation causing the water molecules in the skin vibrate, but the composition of the cells is not influenced. Far infrared rays have the ability to penetrate deeply "up to 1.5 inches" and permeate thoroughly into the hypodermic layer of skin. Thus, it is possible to revitalize cells and tissue by warming the body from the inside. Again, this warming is made possible by the heat energy transfer of the far-infrared radiation. In the far-infrared, however, all tissue absorbs heavily, not just melanin pigment granules.

The sample that was used to expose with $\mathrm{CO}_{2}$ laser is the albino mouse which is a Winstar type, lack of melanin in its skin. Since the rat is albino type, it is failed to tan because a functional tyrosinase is absent from the fully developed epidermal melanin system. Then, the skin burns are the main effect to be considered. The carbon dioxide laser radiation is absorbed very strongly by the skin, with a high absorption coefficient. This means that carbon dioxide laser radiation is deposited in a localized depth of skin and therefore the temperature rises can be higher locally than for the case of visible laser radiation which is transmitted through greater depths of skin. The skin was assumed to be homogeneous, with the thermal properties of water. As the energy of laser increased, burns and damage tissue produced due to absorption of incident laser energy by the natural pigmented materials found in the skin, melanin and hemoglobin. Damage with high keratin content such as the palms was relatively less. The area of the spot of burning skin increased as well as the radiation time increased. The burning spot can be seen clearly as the brown spot and the burning spot spreading into the bigger area after several minutes. The reaction between the radiated tissue and the surrounding tissue 
caused the burning area to spread out. This burning skin is known as the thermal radiation effect where the energy of laser ray is being absorbed and causing the raise on temperature of radiated skin. Chemical reactions always involve a change in energy. Energy is neither created nor destroyed. Energy is absorbed or released in chemical reactions which can be described as endothermic or exothermic reactions. Here, the chemical reactions in which laser energy is absorbed by skin are involved the endothermic process. Energy in the form of heat is required for the reaction to occur.

The normal rat skin (before exposed to laser radiation) at 40 times of magnification is shown in "Figure 3". The scale is " $200 \mu \mathrm{m}$ ", automatically done by the software based on the current magnification of microscope. This skin is cut at the normal cross section during the sectioning process which influenced by the orientation of sample during the embedding process. The epidermis layer of rat skin and the skin melanin is supposed to be in the innermost of epidermis layer. There are approximately six hair follicles with the sebaceous gland at the left side of hair follicles. Each hair follicle is surrounded by the tissue and there is the adipose tissue at the bottom area which is also called as a fat layer.

The exposed skin at 40 times of magnification is shown in "Figure 4". The scale is " $200 \mu \mathrm{m}$ ", also automatically done by the software based on the current magnification of microscope. The different in skin structure compared to before is caused by the different orientation of sample in sectioning. This sectioning is influenced by the orientation of sample during the embedding process. The epidermis layer of rat skin and the skin melanin is supposed to be in the innermost of epidermis layer. All the structure which is the big circle in shape is the hair follicles. Each hair follicle is surrounded by the tissue and there is the adipose tissue at the bottom area which is also called as a fat layer.

The heat effect of laser energy that was absorbed by the skin caused the expanding of empty space between the hair follicles and its surrounding tissue. This phenomenon occurs because of the skin tissue is shrunk as the laser energy being absorbed and heated the water molecules in skin being heated. This endothermic process caused the vaporization of water molecules in exposed skin.

The full view of the normal rat skin before the exposure to carbon dioxide laser radiation is shown in "Figure 5". There is an epidermis layer, a dermis layer and the hypodermis or subcutaneous layer of skin. All the structure in dermis layer "second layer" is the darker than surrounding tissue are the hair follicles. The third and fourth layers are the subcutaneous layer.

The full view of the rat skin after being exposed to carbon dioxide laser radiation is shown in "Figure 6". Besides the expanded of empty space between the hair follicles and its surrounding tissue, the thermal radiation effect also caused the dermis layer separated from the subcutaneous layer. This phenomenon also caused by the shrinkage tissue as the effect of vaporization of water molecules in skin structure.

In the overall view, the skin structure seems to be shrinking after the exposure to carbon dioxide laser. This shrinkage structure caused by the energy of laser absorbs on the soft tissue and makes the water in that tissue heats up and vaporizes. It is proved the fact that during lasing time, water in target tissue absorbs the laser energy, heats up, boils and vaporizes, taking the surrounding tissue with it.

Since the carbon dioxide operates in the far infra red spectral region, it may have the properties of far infrared radiation. A far infrared wave has an ability to remove toxins. Toxic gases may be encapsulated by clusters of water. When a 10 micron far infrared wave is applied to these large water molecules, the water begins to vibrate, which reduces the ion bonds of the atoms which are holding together the molecules of water. As the breakdown of the water molecules occurs, encapsulated gases and other toxic materials are released. Interaction of far infrared radiation with the body also produces nitric oxide gas "NO" by the reaction of the photons of the far infrared on the enzymes, which is useful for body immunization and faster wound healing.

\section{Conclusion}

This paper studied the laser effects on skin melanin. The main objective is to know the effectiveness of laser radiation on the skin melanin. The best way to determine the result is on the facts about the skin and also the expected results according to the published paper that discussed on the studies of radiation and skin. Subsequently, it is closely related with the application of laser in medical field. During preparing this paper, the experiment is carried out to determine the effect of laser radiation on the skin melanin. The type of laser which is selected to be used is depends on the wavelength of output laser which is related to the absorption of that wavelength on the target tissue. It also depends on which laser is available at the school. Then, the sample to be used is decided based on the experiment by other researchers. The color of the sample is changed due to the dose of radiation, time exposure and also the pigment or melanin contains on that sample. Furthermore, the exposed sample is examined on many ways. Therefore, this gives a hint for us to select the way on how to examine the results for data analysis and results.

\section{References}

Aesthetic Laser Concepts. Available from World Wide Web: http://www.aestheticlaserconcepts.com/about.html, (July $22,2007)$. 
Andrews, D.L, Lasers in Chemistry (1986) (New York: Springer-Verlag), pp.15-18 and p.p. 31-35.

Blois, M.C., Quevedo, W.C. et al. (1969), The Biological Effects of Ultraviolet Radiation. (Oxford: Pergamon Press), pp. 325-326.

C. Dierickx, C. et al, (2004), You're Looking Great. Available from World Wide Web: http://youre-looking-great.com/effective_paper.htm, (July 22, 2007).

Grossman, K. Epidermis Anatomy-Skin \& Beauty, Available from World Wide Web:http://dermatology.about.com/od/anatomy/ss/epidermis_2.htm, (July 22, 2007).

Lee, S. Lasers, General Principles and Physics - e-Medicine. Available from World Wide Web: http://www.emedicine.com/ent/topic40.htm, (January 20, 2008) .

Long Beach Animal Hospital. (2007). Available from World Wide web:http://www.lbah.com/laser.htm, (March 24, 2008).

Parks, J. et al, (2002), Effectively Treating Ethnic Skin -Skin \& Aging, Available from World Wide Web: http://www.skinandaging.com/article/576, (July 22, 2007 ).

Table 1. The Thickness of Dermis Layer

\begin{tabular}{|c|c|}
\hline No. & Horizontal distance $(\boldsymbol{\mu m})$ \\
\hline 1 & 979.04 \\
\hline 2 & 997.01 \\
\hline 3 & 1004.49 \\
\hline 4 & 967.07 \\
\hline 5 & 977.54 \\
\hline Average & $\mathbf{9 8 5 . 0 3}$ \\
\hline
\end{tabular}

Table 2. Thickness of epidermis layer

\begin{tabular}{|c|c|}
\hline No. & Thickness $(\boldsymbol{\mu m})$ \\
\hline 1 & 48.58 \\
\hline 2 & 57.61 \\
\hline 3 & 43.52 \\
\hline 4 & 46.98 \\
\hline 5 & 33.64 \\
\hline 6 & 42.18 \\
\hline 7 & 34.17 \\
\hline 8 & 51.71 \\
\hline Average & $\mathbf{4 4 . 8 0}$ \\
\hline
\end{tabular}




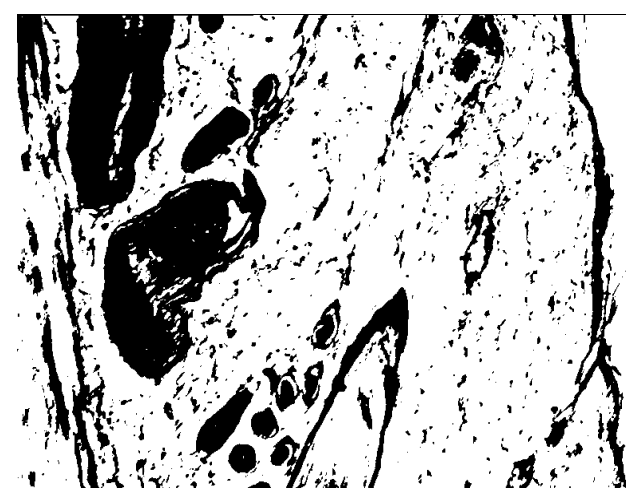

Figure 1. Dermis layer, $40 \mathrm{x}$

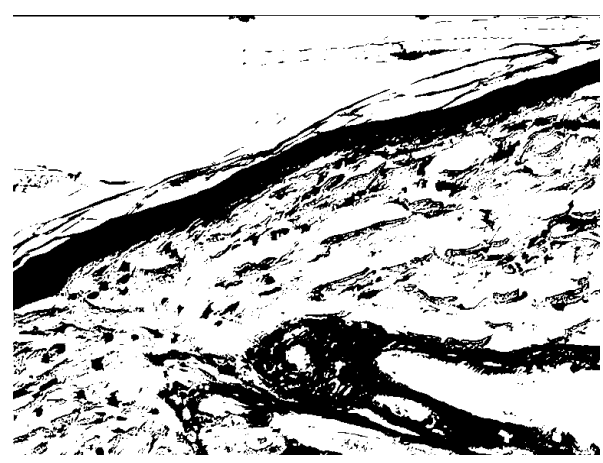

Figure 2. Epidermis layer, $100 \mathrm{x}$.

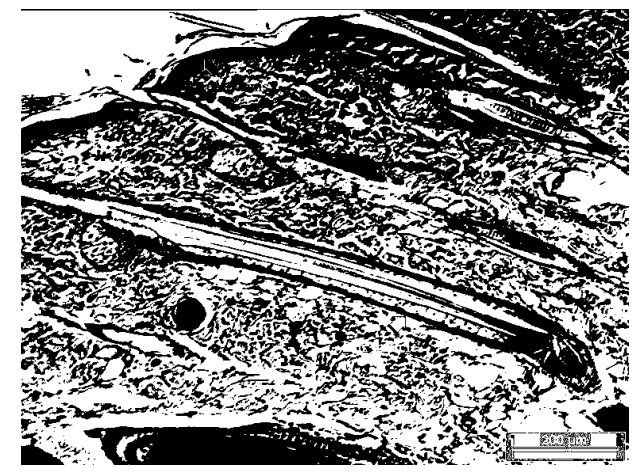

Figure 3. Normal Skin, $40 \mathrm{x}$. 


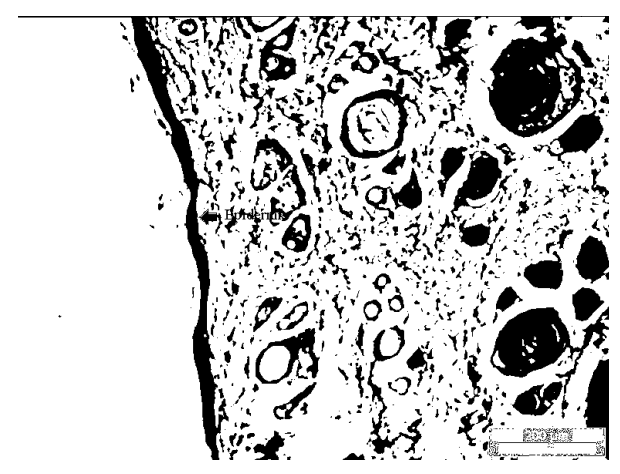

Figure 4. After exposure to $\mathrm{CO}_{2}$ Laser, 40x.

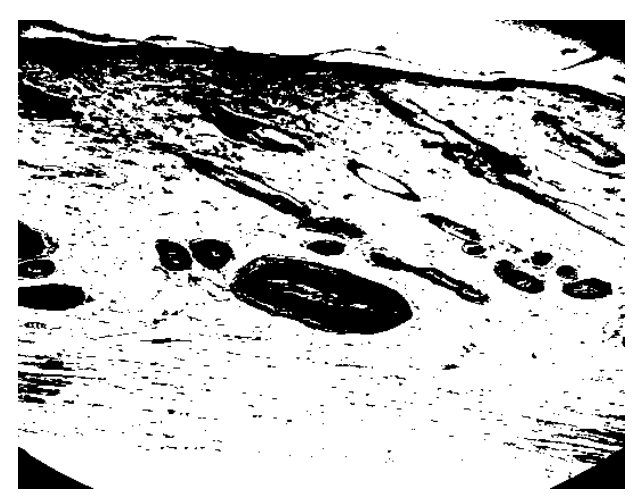

Figure 5. The full view of the normal rat skin

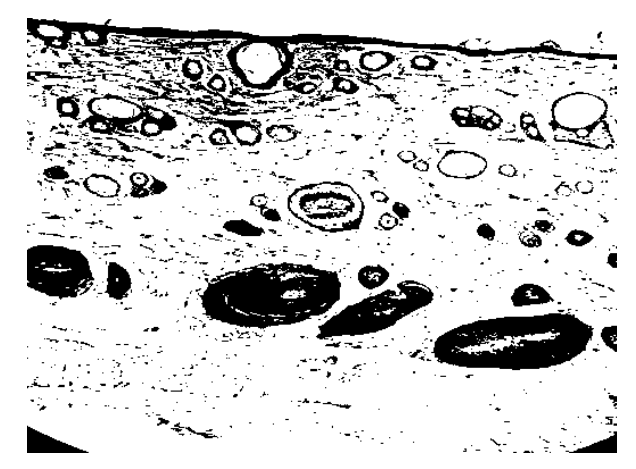

Figure 6. The full view of the exposed rat skin 